引用格式: 李旭, 熊勇清. 新能源汽车“双积分”政策影响的阶段性特征: 经营与环境双重绩效视角 [J]. 资源科学, 2021, 43(1): 1-11. [Li X, Xiong Y Q. Characteristics of the impact of corporate average fuel consumption-new energy vehicle credits (CAFCNEV) mandate on the new energy vehicle industry: From the perspective of business and environmental performances[J]. Resources Science, 2021, 43(1): 1-11. ] DOI: 10.18402/resci.2021.01.01

\title{
新能源汽车“双积分”政策影响的阶段性特征 经营与环境双重绩效视角
}

\author{
李 旭, 熊勇清
}

(中南大学商学院, 长沙 410083)

\begin{abstract}
摘 要: “双积分”政策目的在于促进新能源汽车产业经营绩效与环境绩效的同步增长。以中国新能源乘用车 (整车) 上市公司作为研究样本,将“双积分”政策划分为酝酿期 (2014-2016年)和实施期 (2017-2018年), 从显著 性、敏捷性和稳定性 3 个维度, 分析了“双积分”政策影响下新能源车企经营绩效与环境绩效的动态变化特征。结果 表明: (1)“双积分”政策在酰酿期便提前释放出了积极作用,环境绩效增长更显著、更稳定,经营绩效政策响应更敏 捷; (2) “双积分”政策在实施期的积极作用进一步增强, 经营绩效增长更显著, 环境绩效政策响应更敏捷, 增长也更 稳定。政策制定者在完善和优化“双积分”政策时要善于利用政策信号释放机制，进一步提高和扩大“双积分”政策 的适应性、覆盖面和精准度,把握好政策实施和执行的节奏和力度。
\end{abstract}

关键词: “双积分”政策; 经营绩效; 环境绩效; 新能源汽车;反事实分析

DOI :10.18402/resci.2021.01.01

\section{1 引言}

中国近年来依托政府补贴、税费减免等“补贴 型”政策, 虽然在短期内促进了新能源汽车产销规 模的增长, 但是这种“补贴型”政策缺乏可持续性 ${ }^{[1]}$, 并且引发了部分车企的“谋补骗补”等问题 ${ }^{[2]}$ 。为 此,中国借鉴美国加州地区的零排放汽车积分政策 (Zero Emission Vehicle Mandate, ZEVM) 和欧盟排 放交易体系 (European Union Emission Trading Scheme, EU ETS), 从 2014 年开始酝酿并于 2017 年 9 月正式推出了“乘用车企业平均燃料消耗量与新 能源汽车积分并行管理办法” (简称“双积分”政 策）。“双积分”政策作为中国新能源汽车“补贴退 坡”后的主要承接政策,其目的是促进新能源汽车 产业经营绩效与环境绩效的同步增长, 并建立新能 源汽车产业的长效发展机制 ${ }^{[3]}$ 。积分管理政策尽管
在户籍管理 ${ }^{[4]}$ 、环境保护 ${ }^{[5]}$ 、社会服务 ${ }^{[6]}$ 等公共事务管 理领域被认为是一种有效的政策工具,但是能否促 进新能源汽车产 (企) 业的经营绩效与环境绩效协 同发展, 目前还处于探索过程中。

学术界针对新能源汽车已有的一些产业政策 能否促进经营与环境绩效的协同发展开展了一些 研究 ${ }^{[7-9]}$,但是并没有取得一致性结论 ${ }^{[10]}$ 。究其原因, 一方面是由于产业政策的实施与扩散往往会经历 宣传酝酿、发布实施、优化调整等几个阶段,不同阶 段的作用机理存在差异 ${ }^{[1]}$ 。伴随着政策的阶段性变 化, 新能源汽车企业的经营绩效与环境绩效实际上 也处于动态变化中。另一方面是由于新能源汽车 企业的经营决策往往具有策略性 ${ }^{[12]}$ 。在产业政策酝 酿或实施的不同阶段,新能源车企基于自身利益的 策略性反应将呈现阶段性变化,从而导致不同阶段

收稿日期: 2020-02-09, 修订日期: 2020-10-31

基金项目:国家自然科学基金项目（71874208）。

作者简介: 李旭,男,湖南汉寿人,博士研究生,研究方向为新兴产业与创新战略。E-mail: lixu151601038@163.com

通讯作者:熊勇清,男,江西临川人,教授,研究方向为新兴产业与创新战略。E-mail: xyq@csu.edu.cn 
经营绩效与环境绩效的动态变化。

关于新能源汽车积分管理政策的已有研究也 存在类似争议。国外部分学者认为积分管理政策 (主要针对 ZEV 积分政策) 在促进新能源汽车产业 双重绩效增长过程中具有显著效果, 如 Cristello 等 ${ }^{[13]}$ 的研究发现美国加州 ZEV 积分管理政策体现了环 境保护与产业创新的潜在互补性, 环境绩效与经营 绩效呈现协同增长。Wesseling 等 ${ }^{\left[{ }^{[14]}\right.}$ 研究认为 ZEV 政策能够诱导新能源车企从“防御性”应对向研发 创新和支持规制等 “主动性” 战略转变, 研发专利和 市场规模等经营绩效增长更显著。Sykes 等 ${ }^{[15]}$ 的研 究认为 ZEV 政策的强制性有效避免了技术创新溢 出中 “搭便车”现象, 车企经营绩效中的技术绩效提 升更明显。但是, 也有部分学者认为积分管理政策 在促进新能源汽车双重绩效增长时效果十分有限, 如 Rhode 等 ${ }^{[16]}$ 的研究认为政策制定者可能因为经济 效率、行政可行性、公平和政治可接受性等原因缺 乏制定和执行 ZEV 政策的动机, 环境绩效改善困 难。Wessling ${ }^{[17]}$ 的研究认为最终很难将汽车产业技 术突破或经营绩效增长归因于 ZEV 政策。Dua 等 ${ }^{[18]}$ 的研究认为传统燃油车的促销政策会弱化 ZEV 政 策的效果。Hammond 等 ${ }^{[19]}$ 的研究认为单一的 ZEV 政策以及执行不严格反而导致了环境绩效下降。 由于中国实施“双积分”政策时间不长, 仅有一些学 者针对“双积分”政策实施后汽车制造商生产决

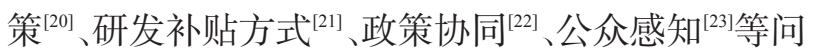
题开展了研究, 关于 “双积分” 政策在促进新能源车 企经营绩效与环境绩效中作用的研究比较少见。

已有研究存在 3 个不足:一, 中国新能源汽车 “双积分”政策虽然是在借鉴美国 ZEV 积分政策和 欧盟排放交易体系基础上形成的,但是中国 “双积 分” 政策的具体内容和相关指标与这些国家的积分 管理政策有所区别,并且中国新能源汽车的技术、 市场等要素也与这些国家和地区存在差异,国外已 有的一些研究结论并不一定完全适用于中国; 二, “双积分”政策实施的目的在于促进新能源汽车产 业经营绩效和环境绩效的协同发展, 已有研究较为 重视经营绩效(包括市场绩效和技术绩效等)方面 的评价与测量, 对于环境绩效等可持续发展维度的 评价关注不够; 三, 产业政策的影响普遍存在“提前
释放期” [24] “双积分”政策在酝酿期便对新能源汽车 产业形成了相当的影响力,已有研究较为重视政策 实施期的影响,对于“双积分”政策酝酿期的影响及 其动态变化过程缺乏足够关注。

为此,本文将中国新能源汽车“双积分”政策的 实施区分为“酝酿期”和“实施期”两个阶段,并以新 能源乘用车(整车)上市公司作为研究样本, 分析中 国新能源车企“经营绩效”和“环境绩效”的动态变 化特征, 以期为进一步提高中国新能源汽车“双积 分”政策的精准性提供决策参考。

\section{2 理论分析与研究假设}

\section{1 “双积分”政策与新能源车企的经营绩效}

技术和市场是影响经营绩效的两个关键要素, “双积分”政策的实施不仅可以促进新能源车企的 技术绩效，同时也有助于促进其市场绩效。一方 面，“双积分”政策中 “企业平均燃料消耗量积分” (Corporate Average Fuel Consumption Credits, CAF$\mathrm{CC})$ 规定了逐年提高平均燃料能耗达标的具体要 求, “新能源汽车积分” (New Energy Vehicle Credits, $\mathrm{NEVC}$ ) 则通过计分系数差异和续航里程门槛明确 了新能源汽车的技术创新方向。相关研究表明,基 于“特定”技术类型和标准的政策规制对于加速技 术创新和形成规模经济等短期经营绩效效果更 好 ${ }^{[25]}$, 因此“双积分”政策对于新能源车企的技术绩 效有着积极的促进作用。另一方面，“双积分”政策 规定了新能源汽车产销量的强制占比，同时结合路 权优先、基础设施建设等鼓励新能源汽车的供需双 侧政策,为新能源车企创造了更好的竞争环境和市 场机会, 有助于促进新能源车企的市场绩效。因 此,可以提出研究假设:

H1: “双积分”政策有助于促进新能源汽车企业 的经营绩效。

H1a: “双积分”政策背景下,新能源车企的技术 绩效将获得提升。

H1b: “双积分”政策背景下, 新能源车企的市场 绩效将获得提升。

\section{2 “双积分”政策与新能源车企的环境绩效}

“双积分”政策不仅具有节能减排的环境贡献 和公共价值, 同时也有利于实现新能源车企的可持 续增长。一方面，“双积分”政策规定了两类汽车产 
销占比和两类积分达标考核要求, 有助于促进节能 与新能源汽车的推广使用和降低汽车尾气温室气 体排放水平。另一方面, “双积分”政策规定了车企 CAFC 负积分可以通过 CAFC 正积分结转或 NEV 正 积分冲抵、“富余的”NEV 正积分可以通过交易获 利, 从而有助于新能源车企的技术创新偏向“环境”[26], 促进新能源车企环境绩效增长。为此, 可以提出研 究假设:

$\mathrm{H} 2$ : “双积分”政策有助于促进新能源汽车企业 的环境绩效。

\section{3 “双积分”政策对新能源车企绩效影响的阶段差} 异性

在政策试点与实施的不同阶段,政策目标和约 束条件决定了相应的制度安排和作用力度,也产生 了不同的政策绩效 ${ }^{[27]}$ 。中国新能源汽车“双积分”政 策从宣传酝酿到发布实施, 对于新能源车企的绩效 影响也必然存在阶段差异性。同时, 不同车企基于 自利的策略反应也会使得政策作用效果在响应速 度 (敏捷性)、显著程度 (显著性)和持续时间 (稳定 性)等主要方面存在差异 ${ }^{[28]}$ 。在“双积分”政策的酝 酿期, 国家相关部门反复征求意见, 实际上也是向 新能源车企释放从“补贴” 向 “积分考核”转变的政 策信号。“主动捕捉”政策信号并提前作出反应, 这 是现代企业经营活动中的重要内容 ${ }^{[29]}$, 因此, “双积 分”政策在酝酿期便对于新能源车企的绩效形成了 实质性影响。具体而言, 随着“双积分”改革信号的 释放,多数新能源车企将会“提前布局”, 扩大研发 投人, 以谋求在新能源汽车 “跑道”上获得技术先发 优势 ${ }^{[30]}$ 。同时也将促进一些新能源车企扩大市场推 广力度, 以建立市场规模优势。在技术进步和规模 性减排的双重作用下, 环境绩效增长更显著 ${ }^{[31]}$ 。因 此, 在 “双积分”政策酝酿期, 新能源车企经营绩效 的政策响应相对更加敏捷, 环境绩效相比经营绩效 增长更显著。在“双积分”政策的实施期,伴随着新 能源汽车财税补贴的加速退坡, 新能源汽车的市场 绩效增长将有所放缓。迫于积分考核压力, 新能源 汽车企业将趋于通过技术创新获得核心竞争力, 由 技术创新而带来的环境绩效增长稳定性将相应增 强 ${ }^{[32]}$ 。因此,可以提出研究假设：
$\mathrm{H} 3$ : 新能源车企绩效在“双积分”政策的酝酿期 和实施期存在差异性。

H3a: “双积分”政策酝酿期,经营绩效响应更敏 捷,环境绩效增长更显著。

H3b: “双积分”政策实施期,经营绩效增长更显 著,环境绩效增长更稳定。

\section{3 研究设计}

\section{1 分析过程与模型构建}

(1)分析过程。由 “基准回归模型分析” 和“反 事实分析检验”两个步骤构成:

步骤一:基准面板向量自回归模型分析。面板 向量自回归模型 (Panel Vector Autoregression, PVAR) 可以分析变量受到另一变量冲击时所表现 出的短期响应和长期变化趋势, 并且能够避免变量 互为因果等内生性问题,被广泛应用到产业政策与 技术绩效、环境规制与可持续增长等政策效果评价 研究中 ${ }^{[33]}$ 。为此, 本文以 PVAR 作为基准模型, 估计 “双积分”政策冲击对新能源车企双重绩效的影响。

步骤二:反事实分析与检验。反事实分析是通 过模拟政策实施前后样本的指定变量之差来估计 政策效果的方法,被认为是实用性较强的政策评价 新范式。本文构建反事实分析框架, 具体做法是： 将步骤一基准模型中“双积分”政策对新能源车企 研发投人的影响系数设定为 0 , 模拟无“双积分”政 策背景下的情形并以此构成反事实检验。通过比 较基准模型与反事实模拟下的脉冲响应函数差异, 评价“双积分”政策的作用效果。

（2）模型构建。构建“双积分”政策影响下新能 源车企双重绩效的 PVAR 分析模型为:

$$
\boldsymbol{Y}_{i t}=\prod+\sum_{p=1}^{n} \prod_{n p} Y_{i(t-p)}+\sum_{p=1}^{n} \prod_{n p} X_{i(t-p)}+\gamma_{i}+\eta_{i}+\mu_{i t}
$$

式中: $\boldsymbol{Y}_{i t}$ 是被解释变量,包括 $[M P, T P, E P]$, 为三维列 向量; $M P$ 和 $T P$ 分别表示 “市场绩效 (Market Performance)”和“技术绩效 (Technical Performance)”, $E P$ 表示“环境绩效(Environmental Performance)”; П为 待估计的参数矩阵; $n$ 表示样本数量; $p$ 为 PVAR 模 型选择的滞后阶数; $X_{i(t-p)}$ 为解释变量, 表示 “双积分” 政策对新能源车企绩效的冲击; $i$ 代表新能源车企; $t$ 为时间; $\gamma_{i}$ 和 $\eta_{i}$ 分别为个体效应和时间效应; $\mu_{i t}$ 为随 
机扰动项且服从期望为 0 、协方差为 $\Phi$ 的独立同分 布。该模型可以进一步分解为:

$$
\begin{gathered}
M P_{i t}=\prod^{+}+\sum_{p=1}^{n} \prod_{n p} M P_{i(t-p)}+\sum_{p=1}^{n} \prod_{n p} X_{i(t-p)}+\gamma_{i}+\eta_{i}+\mu_{i t} \\
T P_{i t}=\prod^{+}+\sum_{p=1}^{n} \prod_{n p} T P_{i(t-p)}+\sum_{p=1}^{n} \prod_{n p} X_{i(t-p)}+\gamma_{i}+\eta_{i}+\mu_{i t} \\
E P_{i t}=\prod^{+} \sum_{p=1}^{n} \prod_{n p} E P_{i(t-p)}+\sum_{p=1}^{n} \prod_{n p} X_{i(t-p)}+\gamma_{i}+\eta_{i}+\mu_{i t}
\end{gathered}
$$

新能源车企经营绩效 (Business Performance, BP) 由模型 (2)和 (3)构成, 新能源车企环境绩效由 模型(4)构成。

(3)变量说明。模型中各变量如下:

(1)被解释变量。 $\boldsymbol{Y}_{i t}$ 为被解释变量,包括新能源 车企经营绩效和环境绩效两个维度变量。在经营 绩效方面, 以“新能源乘用车年产(销)量/乘用车全 年产 (销) 总量” 衡量新能源车企的市场绩效, 以“发 明专利数量” 衡量新能源车企的技术绩效。在环境 绩效方面, 以“环境全要素生产率 $(E A M F P)$ ”衡量新 能源车企节能减排水平和可持续增长方面的环境 绩效 ${ }^{[34]}$ 。

(2)解释变量。“双积分”政策 (CAFC-NEV Mandate, CNM) 为解释变量。“双积分” 政策的两类积分 分值反映了上一年度车企应对 “双积分” 政策规制 的策略意图和行为结果, 工信部从 2013 年以来, 每 年年初都会通过公示纳人考核的新能源车企两类 积分分值,藉此释放“双积分”政策的改革信号。为 此, 本文以“双积分”政策 $(C N M)$ 作为解释变量, 并 以标准化后的上一年度 CAFCC 与 NEVC 之和作为 测量指标。

(3)中介变量。“双积分”政策目标必须通过促进 新能源车企研发创新才能实现政策信号传导。因 此,本文选取“研发投人”作为中介变量, 以研发投
人强度 $(R \& D$ Intensity) 作为测量指标。

(4)控制变量。本文选取企业规模、盈利能力和 所有权性质作为车企异质性控制变量, 以期末总资 产、企业净资产收益率(上期)、虚拟变量 (国有企业 为 1 ; 其他企业为 0$)$ 作为测量指标, 在反事实分析框 架中,增加政府补贴、税费减免作为控制变量, 以政 府补贴/营业收人、(企业支付税费 - 税费返还)/营 业收人作为测量指标。

\section{2 研究样本与数据来源}

(1) 时间窗口及阶段划分。2010年被认为是新 能源汽车产业“元年”,初期以“政府补贴” “税费减 免”等补贴型产业政策为主。从 2014 年起,中国开 始酝酿“双积分”政策并逐步实施“补贴退坡”计 划。尽管“双积分”政策于 2017 年正式公布,但是从 2014年“双积分”政策进人䣲酿阶段,已经对汽车制 造商的经营活动产生了实质性影响。据此,本文将 2014年作为检验“双积分”政策影响效果的分界点， 具体划分为“双积分”政策 酛酿期(2014-2016年) 和实施期(2017-2018年)两个阶段。

(2)研究样本与数据来源。以 2012-2018年期 间中国新能源乘用车(整车)上市公司作为研究样 本,数据主要来源于国家知识产权局数据库、Wind 数据库、国泰安数据库、《节能与新能源汽车年鉴》 （2012-2018年)。

\section{4 结果与分析}

\section{1 模型的稳定性检验}

(1)面板单位根检验与最优滞后阶数。检验结 果如表 1 和表 2 , 采用同根 LLC 检验和异根 Fisher$\mathrm{ADF}$ 检验, 均拒绝存在单位根的原假设, 表明变量 序列平稳。将“双积分”政策变量分别与各维度绩 效变量进行 PVAR 估计,信息准则结果显示市场绩 效和技术绩效最优滞后阶数为 1 阶, 环境绩效最优 滞后阶数为 2 阶。

表 1 面板单位根检验结果

Table 1 Unit root test results of panel data

\begin{tabular}{cccccc}
\hline 检验方法 & $M P$ & $T P$ & $E P$ & $C N M$ & 属性判断 \\
\hline ADF 检验 & $-2.65^{* *}$ & $-0.79 *$ & $-3.13^{* * *}$ & $-2.23^{* *}$ & 平稳( 1 st-d,none $)$ \\
LLC 检验 & $-7.75^{* * *}$ & $-9.79 * * *$ & $-9.99 * * *$ & $-8.97 * * *$ & \\
\hline
\end{tabular}

注: $* * * * * *$ 分别表示该系数在 $1 \% 、 5 \% 、 10 \%$ 的显著水平上显著。下同。 
表 2 最优滞后阶数检验结果

Table 2 Test results of optimal lag order

\begin{tabular}{|c|c|c|c|c|c|c|c|c|c|}
\hline \multirow{2}{*}{ 阶数检验 } & \multicolumn{3}{|c|}{$M P$} & \multicolumn{3}{|c|}{$T P$} & \multicolumn{3}{|c|}{$E P$} \\
\hline & $A I C$ & $B I C$ & $H Q I C$ & $A I C$ & $B I C$ & $H Q I C$ & $A I C$ & $B I C$ & $H Q I C$ \\
\hline 1 & $-9.46^{*}$ & $-8.43 *$ & $-9.05^{*}$ & $0.59 *$ & $1.62 *$ & $0.99 *$ & 3.59 & 4.61 & 3.99 \\
\hline 2 & -8.89 & -7.59 & -8.39 & 1.58 & 2.89 & 2.09 & $1.96^{*}$ & $3.26 *$ & $2.47 *$ \\
\hline 3 & -7.50 & -5.85 & -6.89 & 2.89 & 4.54 & 3.49 & 2.69 & 4.35 & 3.30 \\
\hline
\end{tabular}

(2)PVAR 稳定性检验与 Granger 因果检验。利 用最优滞后阶数进行回归检验 PVAR 的稳定性, 特 征根均小于 1 , 落在单位圆内, 说明 PVAR 模型是稳 定的。进一步进行两两 Granger 因果检验, 均拒绝 原假设, 说明变量之间互为因果关系, 可以进行脉 冲响应分析和方差分解讨论。

\section{2 政策效果的动态性特征分析}

通过 PVAR 模型的广义矩估计 (GMM)、脉冲响 应图和方差分解 3 类数据, 分别考察“双积分”政策 影响下新能源车企经营绩效与环境绩效的显著性、 敏捷性和稳定性动态变化特征,结果分别如表 3 、图 1 和表 4 。

(1)“双积分”政策酝酿期 (2014-2016年)的影 响效果分析

(1)显著性特征分析。“双积分”政策对于新能源 车企的经营绩效和环境绩效均有显著性影响,环境 绩效的增长相对更加显著。表 3 的广义矩估计 $(\mathrm{GMM})$ 结果显示, 在酝酿期, 经营绩效 $(B P)$ 和环境
绩效 $(E P)$ 的模型方程中, 滞后 1 期和滞后 2 期的 “双 积分”政策效应估计系数均为正,表明新能源车企 双重绩效与 “双积分”政策变量呈现同方向变化, “双积分”政策有利于促进新能源车企双重绩效的 增长。其中, 环境绩效 $(E P)$ 模型方程滞后 1 期和 2 期的“双积分”政策效应估计系数分别为 $0.355(P<$ $0.001) 、 0.435(P<0.001)$, 系数值和显著性均优于经 营绩效 $(B P)$ 模型的估计系数,表明“双积分”政策对 环境绩效的促进效果最显著, 且具有逐渐增强的趋 势。经营绩效 $(B P)$ 中市场绩效 $(M P)(P<0.01)$ 比技 术绩效 $(T P)(P<0.05)$ 的政策促进效果更显著。同 时, 市场绩效 $(M P)$ 与技术绩效 $(T P)$ 滞后一期估计 系数为 $0.827(P<0.001) 、 0.694(P<0.001)$, 环境绩效 $(E P)$ 滞后 1 期和 2 期的估计系数为 $0.422(P<0.05)$ 、 0.021 , 估计系数均为正, 表明新能源车企双重绩效 具有自我增强机制,其中市场绩效的自我增强效应 最显著, 技术绩效次之, 新能源车企的经营绩效比 环境绩效具有更明显的自我积累效果。

表 3 “双积分”政策效应系数的 GMM估计结果

Table 3 Generalized method of moments (GMM) estimation result of corporate average fuel consumption-new energy vehicle credits (CAFC-NEV) mandate effect coefficient

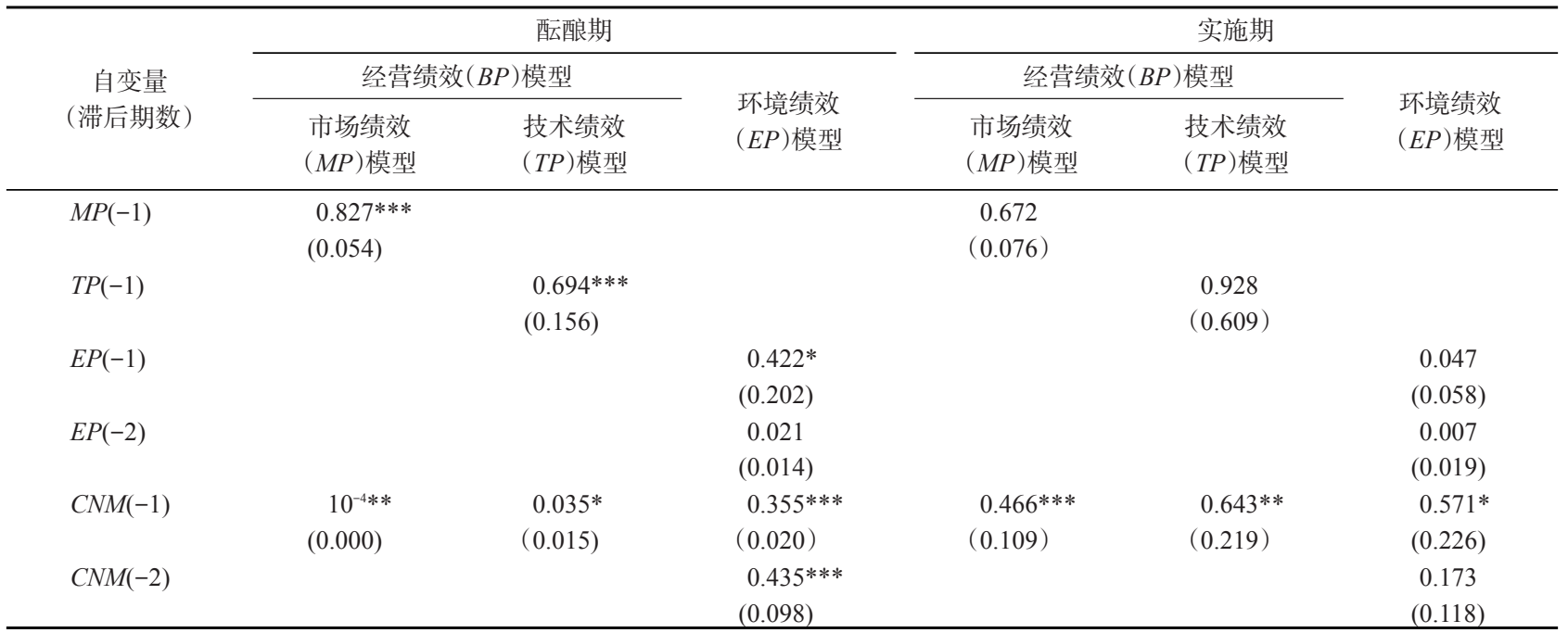



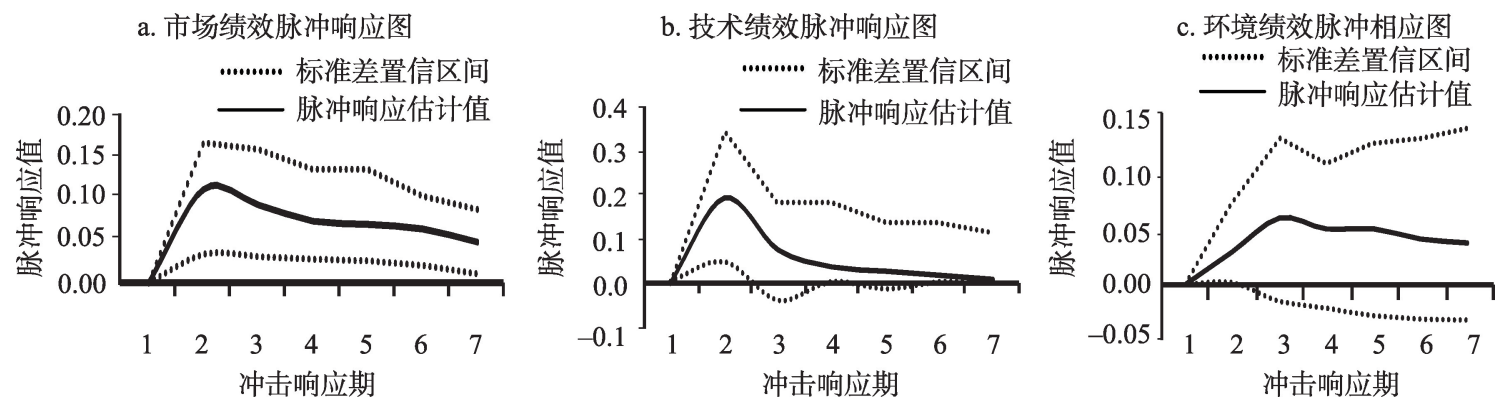

图 1 “双积分”政策冲击下新能源车企双重绩效脉冲响应图

Figure 1 Impulse response chart of business and environmental performance of new energy vehicle enterprises under the impact of the corporate average fuel consumption-new energy vehicle credits (CAFC-NEV) mandate

(2)敏捷性特征分析。新能源车企经营绩效和 环境绩效对“双积分”政策均表现出较高的敏捷性， 经营绩效的反应相对更敏捷。图 1 的脉冲响应分析 结果显示,在酝酿期,受“双积分”政策冲击,市场绩 效 $(M P)$ 和技术绩效 $(T P)$ 有显著的正向脉冲反应, 在滞后 1 期(2015年) 分别达到峰值 0.1 和 0.2 左右, 至酝酿期末 $(2016$ 年)仍维持在 0.07 和 0.03 左右, 政 策响应敏捷, 反应强烈。环境绩效 $(E P)$ 在滞后 2 期 即酝酿期末 $(2016$ 年) 达到峰值 0.06 左右, 政策响应 比较敏捷, 反应比较强烈。表明在“双积分”政策改 革信号的激励下, 新能源车企反应迅速, 双重绩效 提升效果明显,验证了中国情境下产业政策存在提 前释放效应。同时也说明中国新能源车企面对“双 积分”政策的环境规制和创新激励更具主动性, 在 政策酝酿期改革信号传递出来之后 “主动布局”, 双 重绩效增长显著。

(3)稳定性特征分析。新能源车企经营绩效和 环境绩效对“双积分”政策均表现出较高的稳定性，
环境绩效的增长相对更稳定。表 4 的方差分解结果 显示,在酰酿期, “双积分”政策 $(C N M)$ 对新能源车 企双重绩效增长的贡献率同比（以2012 年为基础 期）增长 $2.6 \% 、 17.1 \%$ 和 $16.6 \%$ (2014 年) , 3.4\%、 $17.2 \%$ 和 $37.8 \%$ (2015 年) , $4 \%$ 、 $17.4 \%$ 和 $48.1 \%$ (2016 年),环境绩效相较经营绩效,大幅度增长持续时间 更长, 政策作用稳定; 贡献率环比增速保持同样特 征,其中市场绩效和环境绩效于 2014年同步达到最 大值 $85.7 \%$ 和 $337 \%$, 整个酝酿期，“双积分”政策对 环境绩效贡献率的环比增速始终远高于同年经营 绩效,政策效果更稳定。究其原因,在酝酿期众多 车企已经开始积极布局,谋求新能源汽车“跑道”的 先发优势, 另有不少“造车新势力”跨界进人新能源 汽车行业,创新要素更加丰富; 同时得益于新能源 汽车“补贴型”政策的激励, 节能与新能源汽车实现 了快速推广使用,中国乘用车尾气温室气体排放水 平和总量控制有了明显改善,环境绩效提升更稳 定。实证结果验证研究假设 $\mathrm{H} 3$, 显著支持 $\mathrm{H} 3 \mathrm{a}$ 。

表 4 “双积分”政策促进新能源车企双重绩效增长的方差分解结果

Table 4 Variance decomposition results of the corporate average fuel consumption-new energy vehicle credits (CAFC-NEV) mandate promoting business and environmental performance of new energy vehicle enterprises

\begin{tabular}{|c|c|c|c|c|c|c|c|c|c|c|c|c|c|}
\hline \multirow{3}{*}{\multicolumn{2}{|c|}{ 绩效增长贡献 }} & \multicolumn{8}{|c|}{ 经营绩效 $(B P)$} & \multirow{2}{*}{\multicolumn{4}{|c|}{ 环境绩效 $(E P)$}} \\
\hline & & \multicolumn{4}{|c|}{ 市场绩效 $(M P)$} & \multicolumn{4}{|c|}{ 技术绩效 (TP) } & & & & \\
\hline & & $M P$ & $C N M$ & 环比/\% & 同比/\% & $T P$ & $C N M$ & 环比/\% & 同比/\% & $E P$ & \multirow{2}{*}{$\begin{array}{l}C N M \\
0.000\end{array}$} & \multirow{2}{*}{$\begin{array}{c}\text { 环比/\% } \\
-\end{array}$} & \multirow{2}{*}{$\frac{\text { 同比 } / \%}{-}$} \\
\hline 改革前 & 2012 & 1.000 & 0.000 & - & - & 1.000 & 0.000 & - & - & 1.000 & & & \\
\hline & 2013 & 0.986 & 0.014 & 14.0 & 1.4 & 0.830 & 0.170 & 17.0 & 17.0 & 0.962 & 0.038 & 38.0 & 3.8 \\
\hline \multirow[t]{3}{*}{ 酝酿期 } & 2014 & 0.974 & 0.026 & 85.7 & 2.6 & 0.829 & 0.171 & 0.6 & 17.1 & 0.834 & 0.166 & 337.0 & 16.6 \\
\hline & 2015 & 0.966 & 0.034 & 30.8 & 3.4 & 0.828 & 0.172 & 0.6 & 17.2 & 0.622 & 0.378 & 128.0 & 37.8 \\
\hline & 2016 & 0.960 & 0.040 & 17.6 & 4.0 & 0.826 & 0.174 & 1.2 & 17.4 & 0.519 & 0.481 & 27.2 & 48.1 \\
\hline \multirow[t]{2}{*}{ 实施期 } & 2017 & 0.957 & 0.043 & 7.5 & 4.3 & 0.815 & 0.185 & 6.3 & 18.5 & 0.453 & 0.547 & 13.7 & 54.7 \\
\hline & 2018 & 0.954 & 0.046 & 6.9 & 4.6 & 0.798 & 0.202 & 9.2 & 20.2 & 0.417 & 0.583 & 6.6 & 58.3 \\
\hline
\end{tabular}


（2）“双积分”政策实施期(2017-2018年）

(1)显著性特征分析。“双积分”政策对新能源车 企的经营绩效和环境绩效仍具有正向促进增长, 经 营绩效增长的显著性提高, 环境绩效增长的显著性 下降。表 3 的广义矩估计 (GMM) 结果显示, 在实施 期,经营绩效 $(B P)$ 和环境绩效 $(E P)$ 的模型方程中, 滞后 1 期和滞后 2 期的 “双积分” 政策效应估计系数 仍为正, 且相比酝酿期系数有增大趋势, 说明“双积 分”政策促进新能源车企双重绩效增长的作用逐渐 增强。其中,市场绩效 $(M P)$ 和技术绩效 $(T P)$ 的政 策效应系数分别为 $0.466(P<0.001) 、 0.643(P<$ $0.01)$, 政策作用效果和显著性均有提升, 技术绩效 增长更明显。环境绩效 $(E P)$ 模型方程中 “双积分” 政策 $(C N M)$ 滞后 1 期和 2 期的估计系数分别为 $0.571(P<0.05) 、 0.173$, 相较酝酿期, 滞后 1 期的政策 效应系数虽然有略微增大, 但显著性明显下降, 滞 后 2 期的政策效应系数和显著性同时明显下降。表 明 “双积分” 政策对于新能源汽车产业市场绩效 “量” 的扩张和技术进步 “质” 的提升具有积极促进 作用, 但对环境绩效可持续增长的作用仍有较大提 升空间。

(2)敏捷性特征分析。新能源车企市场绩效和 环境绩效对 “双积分”政策的反应仍然比较敏捷, 技 术绩效的政策响应敏捷性减弱。图 1 的脉冲响应分 析结果显示, 进人实施期, 市场绩效 $(M P)$ 的正向脉 冲反应略有回落, 但仍然维持在 $0.05 \sim 0.10$ 之间, 政 策响应仍然比较敏捷; 技术绩效 $(T P)$ 的正向脉冲反 应下降比较明显, 表明 “双积分”政策技术创新的激 励效果减弱, 车企政策响应敏捷性下降; 环境绩效 $(E P)$ 的正向脉冲反应始终稳定在 0.04 左右, 表明政 策响应敏捷性比较恒定。其原因可能是随着“双积 分” 政策达标规则的优化和补贴加速退坡, 更高水 平的技术创新短期内无法实现更大突破, 实施期的 技术绩效激励效果没有酝酿期明显, 经营绩效增长 放缓。但是得益于节能与新能源汽车产销规模的 持续增长和技术积累, 新能源车企环境绩效对 “双 积分” 政策的响应更敏捷。实证结果证实了研究假 设 $\mathrm{H} 1$ 和 $\mathrm{H} 2$ 。

(3)稳定性特征分析。新能源车企经营绩效和 环境绩效增长趋势依然比较稳定, 环境绩效增长的
稳定性更明显。表 4 的方差分解结果显示, 进人实 施期, “双积分”政策 $(C N M)$ 对新能源车企双重绩效 增长的贡献率同比 (以 2012 年为基础期) 增长 $4.3 \% 、 18.5 \%$ 和 $54.7 \%$ (2017 年 ) , $4.6 \% 、 20.2 \%$ 和 $58.3 \%$ (2018 年), 贡献率同比仍然保持增长但增速 有所放缓,其中, “双积分”政策对环境绩效增长的 贡献率相对更稳定且远高于同年经营绩效; 贡献率 环比增速分别为 $7.5 \% 、 6.3 \%$ 和 13.7\%(2017 年), $6.9 \% 、 9.2 \%$ 和 $6.6 \%$ (2018 年), 其中技术绩效的环比 增速明显增长, 说明进人实施期后, 车企考核压力 增大且技术积累效应显现; 环境绩效的环比增速在 酝酿期高速增长的基础上仍然整体保持稳定增长, 原因可能是得益于中国汽车市场整体需求规模大、 供需双方环保意识增强, 节能与新能源汽车实现了 大规模推广使用, “双积分”政策对车企环境全要素 生产率提升持续有效,环境绩效增长稳定。实证结 果验证研究假设 $\mathrm{H} 3$, 显著支持 $\mathrm{H} 3 \mathrm{~b}$ 。

基于以上分析可知, “双积分”政策在酝酿期便 提前释放出了积极作用，环境绩效增长更显著、更 稳定,经营绩效政策响应更敏捷; “双积分”政策在 实施期的积极作用进一步增强, 经营绩效增长更显 著,环境绩效政策响应更敏捷,增长也更稳定。在 “双积分”政策背景下,新能源车企经营与环境双重 绩效具有阶段变动特征, 政策效果定性总结如表 5 。

\section{3 政策效果的反事实检验分析}

本文运用反事实分析法检验在没有受到“双积 分”政策影响的情形下, 即模拟在继续实施原有强 度的补贴型政策情形下, 新能源车企经营和环境双 重绩效的变化特征,构成反事实检验以判断前述因 果推断的可信性。具体做法是:令基准模型中 “双 积分”政策影响研发投人强度的同期系数和滞后系 数为零, 同时保持其他方程的估计系数不变, 重新 估计模型 1 。结合图 1 ,两阶段脉冲响应拟合结果如 图2 所示。

（1）“双积分”政策改革、酝酿与实施的时间节 点把控比较准确, 与 “补贴型”产业政策形成了较好 的政策合力与衔接。如图 2,市场绩效 $(M P)$ 与环境 绩效 $(E P)$ 反事实值与实际值的脉冲响应图在主要 拐点的趋势拟合度较好, 酝酿期增长效果比较显 著, 分别在滞后 1 期和滞后 2 期达到峰值, 反映出 
资源科 学

表 5 新能源汽车“双积分”政策对车企双重绩效影响的阶段性特征

Table 5 Characteristics of corporate average fuel consumption-new energy vehicle credits (CAFC-NEV) mandate promoting business and environmental performance of new energy vehicle enterprises in different stages

\begin{tabular}{|c|c|c|c|c|c|}
\hline \multirow{3}{*}{$\begin{array}{c}\text { “双积分”政策阶段 } \\
\text { 酝酿期 }\end{array}$} & \multirow{2}{*}{\multicolumn{2}{|c|}{ 绩效分析维度 }} & \multicolumn{3}{|c|}{ 政策效果等级 } \\
\hline & & & \multirow{2}{*}{$\frac{\text { 敏捷性 }}{\star \star \star}$} & \multirow{2}{*}{$\frac{\text { 显著性 }}{\star \star}$} & \multirow{2}{*}{$\frac{\text { 稳定性 }}{\star \star}$} \\
\hline & 经营绩效 & 市场绩效 & & & \\
\hline & & 技术绩效 & $\star \star \star$ & $\star \star$ & $\star$ \\
\hline & 环境绩效 & & $\star \star$ & $\star \star \star$ & $\star \star \star$ \\
\hline \multirow[t]{3}{*}{ 实施期 } & 经营绩效 & 市场绩效 & $\star \star$ & $\star \star \star$ & $\star \star$ \\
\hline & & 技术绩效 & $\star$ & $\star \star$ & $\star \star$ \\
\hline & 环境绩效 & & $\star \star \star$ & $\star \star$ & $\star \star \star$ \\
\hline
\end{tabular}

a. 市场绩效脉冲响应拟合图

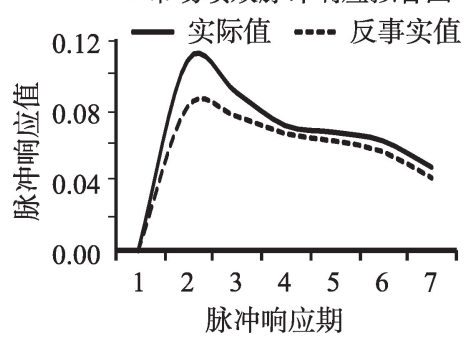

b. 技术绩效脉冲响应拟合图

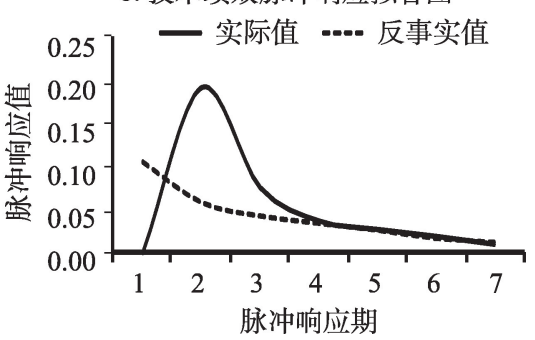

c. 环境绩效脉冲响应拟合图

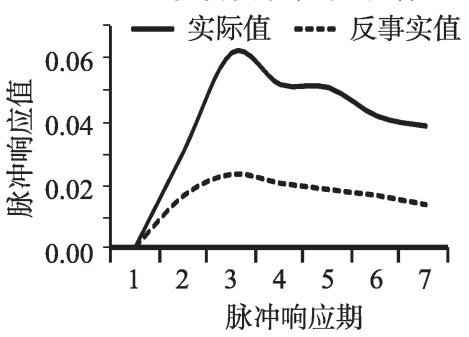

图 2 反事实分析脉冲响应拟合图

Figure 2 Counterfactual analysis pulse response fitting diagram

“双积分”政策改革的时间点和信号传递比较准确， 能够和政府补贴、税费减免等形成较好政策合力。 技术绩效 $(T P)$ 反事实值与实际值的脉冲响应图在 主要拐点的趋势呈现 “反向” 变化, 其中, 反事实值 的趋势线整体下降,表明政府补贴、税费减免等政 策对新能源车企技术绩效虽然具有正向促进作用， 但是长期作用效果下降明显。实际值的趋势线显 示“双积分”政策的确起到了政策衔接作用, 在酝酿 期对技术绩效的促进效果已经显现且达到峰值, 表 明积分管理工具基于技术方向的诱导和技术标准 的强制在短期内激励了车企的技术绩效。进人实 施期, 反事实值与实际值均呈现倒 U 型, 政策合力 效果显著下降。一方面可能是因为补贴退坡加速 抑制了车企继续扩大推广新能源汽车的动机, 另一 方面也因为中国汽车消费规模的整体下滑趋势弱 化了市场拉力的作用。

(2) “双积分” 政策背景下, 新能源车企市场绩 效的政策反应最敏捷,技术绩效的政策促进效果最 显著, 环境绩效的政策增长效果最稳定。如图 2 , 反 事实值脉冲响应峰值分别是市场绩效 $(M P)$ 为 0.08 , 技术绩效 $(T P)$ 为 0.10 , 环境绩效 $(E P)$ 为 0.02 , 低于
实际值的 $0.10 、 0.20 、 0.06$ 。实际值脉冲响应趋势线 整体高于反事实值，说明“双积分”政策有利于促进 新能源车企双重绩效增长。其中,市场绩效 $(M P)$ 实 际值比反事实值的趋势线在酝酿期更加陡峭, 表明 “双积分”政策促进新能源车企调整产品结构,扩大 新能源汽车投放和使用具有更为明显的效果。技 术绩效 $(T P)$ 的峰值差值最大为 0.10 , 表明 “双积分” 政策促进新能源车企技术创新的政策信号传递精 准,在“补占型”产业政策逐步退坡阶段,基本实现 了有效衔接,激励了新能源车企的技术绩效增长。 环境绩效 $(E P)$ 的增长相对滞后, 但整体趋势比较稳 定,正向增长水平始终维持在 0.02 左右, 表明“双积 分”政策试图通过缓解能源和环境双重困境, 汽车 产业环境全要素生产率提高的可持续增长机制逐 渐形成。

\section{5 结论}

本文将“双积分”政策区分为酝酿期和实施期 两个阶段,利用面板向量自回归模型,分析了“双积 分”政策对新能源车企经营和环境双重绩效影响的 动态变化特征,并运用反事实分析法开展了对照检 验。主要结论如下: 
(1) “双积分”政策在酝酿期, 便对于新能源车 企 “提前释放” 出了积极作用。环境绩效的政策作 用效果更显著、更稳定, 经营绩效的政策响应更敏 捷。这表明 “双积分”政策在酝酿期便充分显示了 新能源汽车产业政策由财税补贴向市场导向转型 的积极信号, 对于分阶段缓解 “补贴退坡”压力, 促 进新能源汽车产业发展的内生动力起到了积极的 作用。中国新能源汽车产业政策的制定和实施应 该善于利用酝酿期的信号释放机制, 推动新能源汽 车产业稳定有序发展。

(2) “双积分”政策在实施期, 在促进新能源车 企经营与环境双重绩效过程中的作用进一步增 强。经营绩效的政策作用效果更显著, 环境绩效的 政策响应更敏捷,政策作用效果更稳定。这表明随 着“双积分”政策的正式实施, 新能源汽车产业升级 进人深度调整阶段。中国新能源汽车产业政策实 施和优化需要充分考虑政策“阶段性”,精准把握新 阶段、新特征和变化机理, 强化落实措施, 促进新能 源车企经营与环境双重绩效由阶段性快速增长向 长期稳定增长转变。

（3）反事实检验显示，“双积分”政策改革、酝酿 与实施的时间节点把控比较准确, 与 “补占型”产业 政策形成了高质量适配。新能源车企市场绩效的 政策反应最敏捷,技术绩效的政策促进效果最显 著,环境绩效的政策增长效果最稳定。表明“双积 分”政策对于促进新能源汽车的大规模推广使用, 增强产业发展动力, 强化车企创新驱动等具有积极 作用。下一阶段,应进一步提高和扩大“双积分”政 策的适应性、覆盖面和精准度, 把握好政策实施和 执行的力度和节奏, 建立促进新能源汽车发展的长 效机制。

\section{参考文献(References):}

[1] 白雪洁, 孟辉. 新兴产业、政策支持与激励约束缺失: 以新能源 汽车产业为例[J]. 经济学家, 2018, (1): 50-60. [Bai X J, Meng H. Emerging industries, policy support, and lack of incentives and constraints: Taking the new energy automobile industry as an example[J]. Economist, 2018, (1): 50-60.]

[2] 周亚虹, 蒲余路, 陈诗一, 等. 政府扶持与新型产业发展: 以新 能源为例[J]. 经济研究, 2015, (6): 147-161. [Zhou Y H, Pu Y L, Chen S Y, et al. Government support and development of emerging industries: A new energy industry survey[J]. Economic Research Journal, 2015, (6): 147-161.]

[3] 中华人民共和国工业和信息化部, 财政部, 商务部, 等. 乘用车 企业平均燃料消耗量与新能源汽车积分并行管理办法 $[\mathrm{EB} /$ OL]. (2017- 09- 27) [2020- 02- 09]. http://www.gov.cn/xinwen/ 2017-09/28/content_5228217.htm. [Ministry of Industry and Information Technology of the People's Republic of China, Ministry of Finance, Ministry of Commerce, et al. Measures for Parallel Management of Average Fuel Consumption and New Energy Vehicle Credits of Passenger Vehicle Enterprises[EB/OL]. (2017-0927) [2020- 02- 09]. http://www.gov.cn/xinwen/2017- 09/28/content_5228217.htm.]

[4] 李晓飞. 户籍分割、资源错配与地方包容型政府的置换式治理 [J]. 公共管理学报, 2019, 16(1): 16-28. [Li X F. Household division, resource misallocation and the substituted governance of local inclusive state[J]. Journal of Public Management, 2019, 16(1): $16-28$.

[ 5 ] Mathews J A. How carbon credits could drive the emergence of renewable energies[J]. Energy Policy, 2008, 36(10): 3633-3639.

[6] 钱雪亚, 胡琼, 苏东再, 等. 公共服务享有、居住证积分与农民 工市民化观察 $[J]$. 中国经济问题, 2017, (5): 47-57. [Qian X Y, $\mathrm{Hu}$ Q, Su D R, et al. Public service right, residential permit points and measurement on rural migrant citizenization[J]. China Economic Studies, 2017, (5): 47-57.]

[7] 杨姗姗, 肖尧. 新能源汽车使用补贴政策的经济环境宏观效应: 基于 CGE 模型测度分析[J]. 统计与信息论坛, 2020, 35(6): 115121. [Yang S S, Xiao R. The economic and environmental macroeffect of subsidy policies for new energy vehicles: Based on the simulation of CGE model[J]. Statistics \& Information Forum, 2020, 35 (6): 115-121. ]

[ 8 ] Bergek A, Berggren C. The impact of environmental policy instruments on innovation: A review of energy and automotive industry studies[J]. Ecological Economics, 2014, 106(1): 112-123.

[9] 何文韬. 新能源汽车产业的政策保护与利基构建: 基于多层次 产业演化的分析[J]. 科研管理, 2019, 40(9): 139-148. [He W T. Policy protection and niches construction in the new energy vehicles industry: An analysis based on a multi-level industry evolution[J].Science Research Management, 2019, 40(9): 139-148.]

[10] 曹霞, 邢泽宇, 张路蓬. 政府规制下新能源汽车产业发展的演化 博弯分析[J]. 管理评论, 2018, 30(9): 82-96. [Cao X, Xing Z Y, Zhang L P. An evolutionary game analysis of new energy vehicle industry development under government regulations[J].Management Review, 2018, 30(9): 82-96. ]

[11] 周迪, 周丰年, 王雪芹. 低碳试点政策对城市碳排放绩效的影响 评估及机制分析[J]. 资源科学, 2019, 41(3):546-556. [Zhou D, Zhou F N, Wang X Q.Impact of low-carbon pilot policy on the performance of urban carbon emissions and its mechanism[J].Resources Science, 2019, 41(3): 546-556. 
[12] 张奇, 李曜明, 唐岩岩, 等. 新能源汽车“双积分”政策对生产商 策略和社会福利影响研究[J]. 系统工程理论与实践, 2020, 40 (1): 150-169. [Zhang Q, Li Y M, Tang Y Y, et al. Impact of dualcredit policy on automakers strategies and social welfare[J]. Systems Engineering-Theory \& Practice, 2020, 40(1): 150-169.]

[13] Cristello N, Kim I Y. Multidisciplinary design optimization of a zero-emission vehicle chassis considering crash worthiness and hydro form ability[J]. Journal of Automobile Engineering, 2013, 221 (5): 511-526.

[14] Wesseling J H, Niesten E M M I, Faber J, et al. Business strategies of incumbents in the market for electric vehicles: Opportunities and incentives for sustainable innovation[J]. Business Strategy and the Environment, 2015, 24(6): 518-531.

[15] Sykes M, Axsen J. No free ride to zero-emissions: Simulating a region s need to implement its own zero-emissions vehicle (ZEV) mandate to achieve 2050 GHG targets[J]. Energy Policy, 2017, 110 (11): 447-460.

[16] Rhodes E, Jaccard M. A tale of two climate policies: Political economy of british columbia's carbon tax and clean electricity standard [J]. Canadian Public Policy, 2013, 39(2): 37-51.

[17] Joeri H. Wesseling. Explaining variance in national electric vehicle policies[J]. Environmental Innovation and Societal Transitions, 2016, 21(12): 28-38.

[18] Dua R, White K, Lindland R, et al. Understanding potential for battery electric vehicle adoption using large-scale consumer profile data[J]. Energy Reports, 2019, (5): 515-524

[19] Hammond W, Axsen J, Kjeang E, et al. How to slash greenhouse gas emissions in the freight sector: Policy insights from a technology-adoption model of Canada[J]. Energy Policy, 2020, (137): 111093.

[20] 程永伟, 穆东. 双积分制下汽车生产商生产决策优化[J]. 系统工 程理论与实践, 2018, 38(11): 2817-2830. [Cheng Yongwei, Mu Dong. Optimal production decision of vehicle manufacturer based on double-score system[J]. Systems Engineering-Theory \& Practice, 2018, 38(11): 2817-2830.]

[21] 郑吉川, 赵华, 李志国. 双积分政策下新能源汽车产业研发补贴 研究[J]. 科研管理, 2019, 40(2): 126-133. [Zheng J C, Zhao H, Li Z G. A research on new energy vehicle industry R\&D subsidy under the policy of “double credits" [J]. Science Research Management, 2019, 40(2): 126-133.]

[22] 刘宏笪, 孙华平, 张茜. 中国新能源汽车产业政策演化及执行阻 滞分析: 兼论双积分政策的协同实施[J]. 管理现代化, 2019, 39 (4):41-46. [Liu H D, Sun H P, Zhang Q. Analysis on policy evolution and execution retardation of China's new energy vehicle industry: Also on the coordinated implementation of the doublepoint policy[J]. Modernization of Management, 2019, 39(4): 4146.]

[23] 黄鲁成, 王小丽, 吴菲菲, 等. 基于网络信息挖掘的创新政策公 众感知研究: 以新能源汽车政策为例[J]. 科学学与科学技术管
理, 2019, 40(6): 21-36. [Huang L C, Wang X L, Wu F F, et al. Research on public perception of innovation policy based on network information mining: A case study on new energy vehicle policy [J]. Science of Science and Management of S\&T, 2019, 40(6): 21-36.]

[24] 徐乐, 赵领娣. 重点产业政策的新能源技术创新效应研究[J]. 资 源科学, 2019, 41(1):115-133. [Xu L, Zhao L D. New energy technology innovation effect of key industrial policy[J]. Resources Science, 2019, 41(1): 113-131.]

[25] Sierzchula W, Bakker S, Maat K, et al. Technological diversity of emerging eco-innovations: A case study of the automobile industry [J]. Journal of Cleaner Production, 2012, 37: 211-220.

[26] Greaker M, Heggedal T R, Rosendahl K E. Environmental policy and the direction of technical change[J]. Scandinavian Journal of Economics, 2018, 120(4): 1100-1138.

[27] 梅赐琪, 汪笑男, 廖露, 等. 政策试点的特征: 基于《人民日报》 1992-2003 年试点报道的研究[J]. 公共行政评论, 2015, 8(3): 824. [Mei C Q, Wang X N, Liao L, et al. Patterns of "Experimentation Point”: Evidence from People's Daily's 1992-2003 reports on policy experimentation point[J]. Journal of Public Administration, 2015, 8(3): 8-24.]

[28] 王丽霞, 陈新国, 姚西龙, 等.我国工业企业对环境规制政策的 响应度研究[J]. 中国软科学, 2017,(10): 143-152. [Wang L X, Chen X G, Yao X L, et al. Industrial enterprises' response to environmental regulation policy in China[J]. China Soft Science, 2017, (10): 143-152.]

[29] 熊航, 静峥, 展进涛. 不同环境规制政策对中国规模以上工业企 业技术创新的影响[J]. 资源科学, 2020, 42(7): 1348-1360.

[Xiong H, Jing Z, Zhan J T. Impact of different environmental regulatory tools on technological innovation of Chinese industrial enterprises above designated size[J]. Resources Science, 2020, 42(7): $1348-1360$.

[30] Porter M E, Linde C V D. Toward a new conception of the environment- competitiveness relationship[J]. Journal of Economic Perspectives, 1995, 9(4): 97-118.

[31] Peuckert J. What shapes the impact of environmental regulation on competitiveness? Evidence from executive opinion surveys[J]. Environmental Innovation and Societal Transitions, 2014, 10: 7794.

[32] Oliver C, Holzinger I. The effectiveness of strategic political management: A dynamic capabilities framework[J]. The Academy of Management Review, 2008, 33(2): 496-520.

[33] 黄庆华, 胡江峰, 陈习定. 环境规制与绿色全要素生产率: 两难 还是双赢?[J]. 中国人口・资源与环境, 2018, 28(11): 140-149. [Huang Q H, Hu J F, Chen X D. Environmental regulation and green total factor productivity: Dilemma or win- win?[J]. China Population, Resources and Environment, 2018, 28(11): 140-149.]

[34] Antonakakis N, Chatziantoniou I, Filis G, et al. Energy consumption, $\mathrm{CO}_{2}$ emissions, and economic growth: An ethical dilemma[J]. Renewable and Sustainable Energy Reviews, 2017, 68: 808-824. 


\title{
Characteristics of the impact of corporate average fuel consumption-new energy vehicle credits (CAFC-NEV) mandate on the new energy vehicle industry: From the perspective of business and environmental performances
}

\author{
LI Xu, XIONG Yongqing
}

(School of Business, Central South University, Changsha 410083, China)

\begin{abstract}
To promote the synchronous growth of business performance and environmental performance, the corporate average fuel consumption-new energy vehicle credits (CAFC-NEV) mandate was implemented in the new energy vehicle industry. The implementation can be divided into two stages: the "brewing period" (2014-2016) and the implementation period (2017-2018). Using new energy passenger vehicles as examples, this study examined the dynamic change of business performance and environmental performance of new energy vehicle enterprises from three dimensions significance, agility, and stability. The results show that: (1) The CAFC-NEV mandate had shown its positive effects during the brewing period, in which environmental performance growth was more significant and stable, and business performance responded more quickly to the policy; (2) The positive effects of the brewing period were further enhanced in the implementation period. The growth of business performance is more significant, and the environmental performance policy response is more agile and the growth is more stable. Accordingly, the CAFCNEV mandate needs to further improve its adaptability, coverage, and precision and control the pace and strength of implementation by taking advantage of the policy signal release mechanism.
\end{abstract}

Key words : corporate average fuel consumption-new energy vehicle credits (CAFC-NEV) mandate; business performance; environmental performance; new energy vehicle; counterfactual analysis 